\title{
Adsorption and melting of hydrogen in potassium-intercalated graphite
}

\author{
J. J. Purewal, * J. B. Keith, C. C. Ahn, and B. Fultz \\ W. M. Keck Laboratory, California Institute of Technology, 138-78, Pasadena, California 91125, USA \\ C. M. Brown and M. Tyagi ${ }^{\dagger}$ \\ NIST Center for Neutron Research, 100 Bureau Drive, Gaithersburg, Maryland 20899, USA \\ (Received 17 October 2008; revised manuscript received 21 January 2009; published 27 February 2009)
}

\begin{abstract}
Volumetric adsorption and quasielastic neutron scattering are used to study the diffusion and thermodynamics of sorbed $\mathrm{H}_{2}$ in the graphite intercalation compound $\mathrm{KC}_{24}$. A sorption enthalpy of $8.5 \mathrm{~kJ} / \mathrm{mol}$ at zero coverage is determined from $\mathrm{H}_{2}$ adsorption isotherms. From measurements of total elastic-neutron-scattering intensity as a function of temperature, a melting transition of the $\mathrm{H}_{2}$ adsorbate is observed at $35 \mathrm{~K}$ for $\mathrm{KC}_{24}\left(\mathrm{H}_{2}\right)_{1}$. Quasielastic-neutron-scattering (QENS) spectra reveal distinct slow- and fast- $\mathrm{H}_{2}$-diffusion processes which exist simultaneously at temperatures above the transition point. The temperature dependence of the characteristic diffusion times follows an Arrhenius relation $\tau=\tau_{0} \exp \left(E_{a} / T\right)$, where $\tau_{0}^{\text {fast }}=1.0 \pm 0.1 \mathrm{ps}$, $\tau_{0}^{\text {slow }}=21 \pm 2 \mathrm{ps}, E_{a}^{\text {fast }}=156 \pm 5 \mathrm{~K}$, and $E_{a}^{\text {slow }}=189 \pm 5 \mathrm{~K}$. The fast-diffusion process is attributable to individual motions of $\mathrm{H}_{2}$ molecules in a static potassium structure, and the slow-diffusion process could be attributable to fluctuations in $\mathrm{H}_{2}$ particle density correlated with jumps of potassium atoms. The QENS spectra at low $Q$ are used to discuss the dimensionality of the diffusion process.
\end{abstract}

DOI: 10.1103/PhysRevB.79.054305

PACS number(s): 34.35.+a, 78.70.Nx, 82.75.Jn

\section{INTRODUCTION}

Adsorption of hydrogen $\left(\mathrm{H}_{2}\right)$ gas on carbon surfaces is due to weak London dispersion forces with characteristic energies between 4 and $6 \mathrm{~kJ} / \mathrm{mol}$. $^{1,2}$ This weak interaction limits adsorption to cryogenic temperatures, a drawback for gas storage technologies. Adsorption enthalpies near $15 \mathrm{~kJ} / \mathrm{mol}$ are estimated to optimize $\mathrm{H}_{2}$ storage and delivery at room temperature under moderate pressures. 1,3 "Enhanced physisorption" has been proposed ${ }^{3}$ as a method for increasing sorption enthalpies by inserting alkali-metal dopants into optimally spaced graphene layers.

The second-stage potassium-graphite-intercalation compound, commonly denoted $\mathrm{KC}_{24}$, is one of the few of such systems that can be readily synthesized. The ideal $\mathrm{KC}_{24}$ structure consists of a potassium intercalate layer between every second pair of graphene sheets, as depicted in Fig. 1(b). After the potassium intercalation, the center-to-center interplanar spacing increases from 3.35 to $5.40 \AA$, large enough to reversibly adsorb $\mathrm{H}_{2}$ at low temperatures. 4,5 The spacing increases further to $5.6 \AA$ following $\mathrm{H}_{2}$ adsorption, which provides a gallery of only approximately $2.2 \AA$ when the van der Waals radii of the basal plane $\mathrm{C}$ atoms are taken into account. Because the sorbed $\mathrm{H}_{2}$ molecules are assumed not to cross through the graphite basal planes, their diffusion behavior is considered two dimensional (2D) in a first approximation. The reported $\mathrm{H}_{2}$ adsorption enthalpy in $\mathrm{KC}_{24}$ is $8.4 \mathrm{~kJ} / \mathrm{mol}$, about twice as large as for adsorption on bare graphite. ${ }^{6}$ Charge density surrounding the potassium atoms is thought to be responsible for the large adsorption enthalpy. ${ }^{7}$ This is consistent with a number of computational studies that report an enhancement (by a factor of about 2) of the physisorption energy for $\mathrm{H}_{2}$ interacting with an alkali-doped graphene surface, due largely to the large electron density on the surface surrounding the alkali impurity. ${ }^{8,9}$ Backdonation of electrons from carbon $\pi$ bonds to the $\mathrm{H}_{2}$ molecules is thought to be very small, ${ }^{10}$ indicating that the adsorption mechanism is almost entirely physisorption.
The in-plane potassium structure at low temperatures is not fully understood at present. Single-crystal X-raydiffraction (XRD) studies have shown plausible agreement with a structure consisting of an incommensurate closepacked potassium monolayer which is rotated with respect to the graphite and relaxed into the nearest hexagon centers. ${ }^{11,12}$ In $\mathrm{RbC}_{24}$ and $\mathrm{CsC}_{24}$, however, the low-temperature in-plane structure is thought to consist of locally commensurate $\sqrt{7}$ $\times \sqrt{7} R 19.11^{\circ}$ patches surrounded by domain walls. ${ }^{13,14}$ This $\sqrt{7} \times \sqrt{7}$ structure has been the basis of most $a b$ initio studies of $\mathrm{H}_{2} / M \mathrm{C}_{24}$ systems, and is illustrated in Fig. 1(a). The sorption site is presumably at the center of the trigonal subunit cell (blue square), since filling all of these sites would result in a 2:1 molar ratio of dihydrogen to potassium. Quantum delocalization of the $\mathrm{H}_{2}$ center of mass over the three neighboring sites (red triangles) has also been proposed. ${ }^{15}$

A number of inelastic-neutron-scattering experiments have been performed on $\mathrm{H}_{2} / M \mathrm{C}_{24}(M=\mathrm{K}, \mathrm{Rb}, \mathrm{Cs}),{ }^{15-19}$ which identify rotational tunneling peaks on the order of 1

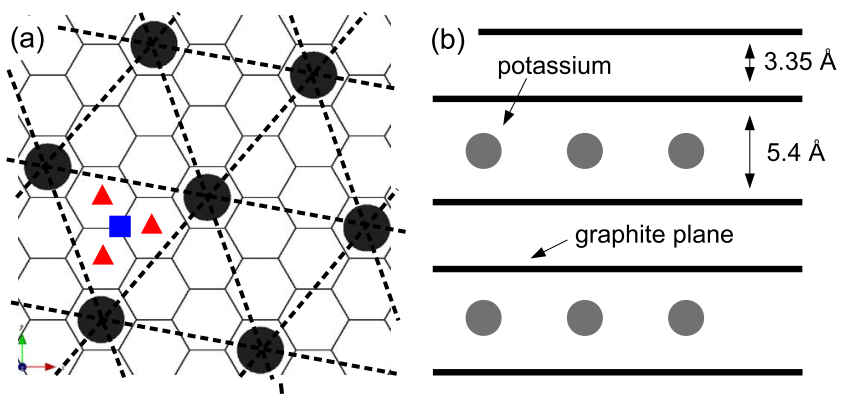

FIG. 1. (Color online) (a) The $\sqrt{7} \times \sqrt{7} R 19.11^{\circ}$ structure, frequently used as an approximation of the low-temperature in-plane arrangement of $\mathrm{KC}_{24}$. This model has a stoichiometry of $\mathrm{KC}_{28}$. The sorption site (blue square) and neighboring sites (red triangles) are depicted for a single trigonal subunit cell. (b) Schematic side view of a stage-2 alkali-metal graphite intercalation compound. 
meV. These low-energy peaks originate from the splitting of rotational energy levels of a hindered $\mathrm{H}_{2}$ quantum rotor, indicating exceptionally strong interactions between $\mathrm{H}_{2}$ and the host material. However, to date there have been no comprehensive studies of $\mathrm{H}_{2}$ diffusivity and phase behavior in $\mathrm{KC}_{24}$. Previous studies of $\mathrm{H}_{2}$ diffusion in zeolites and carbon nanostructures have reported thermally activated liquidlike diffusion, ${ }^{20-23}$ but behavior in the ternary hydrogenpotassium-graphite intercalation compound may be more complicated. The combination of a two-dimensional geometry and a strong sorption potential could create interesting phases of the condensed hydrogen. In the present work, detailed quasielastic-neutron-scattering (QENS) spectra of $\mathrm{KC}_{24}\left(\mathrm{H}_{2}\right)_{1}$ are presented. The temperature dependence of the elastic intensity is used to study the solid-to-fluid phase transition of the $\mathrm{H}_{2}$ adsorbate. Characteristic diffusion times are determined from the quasielastic linewidths of the experimental neutron-scattering data in the limit of large $Q$. The presence of two distinct diffusion processes with different time scales is evident from the experimental spectra. Low- $Q$ experimental spectra are fitted to both two-dimensional and three-dimensional random-walk diffusion models to investigate the geometry of the $\mathrm{H}_{2} / \mathrm{KC}_{24}$ system. In addition, volumetric $\mathrm{H}_{2}$ adsorption measurements are used to verify the enthalpy and capacity of $\mathrm{H}_{2}$ physisorption at $77 \mathrm{~K}$.

\section{METHODS}

The starting materials for $\mathrm{KC}_{24}$ synthesis were purified natural flake graphite (Superior Graphite Co., 99.95-99.99\% purity, 50 mesh) and potassium metal (Alfa Aesar, 99.9\%). The graphite was first outgassed under vacuum at $473 \mathrm{~K}$. Stoichiometric amounts of graphite and potassium (24:1 molar ratio) were weighed out inside an argon glovebox and transferred to a glass tube, which was then connected to a Teflon-sealed stopcock and closed off. The glass tube was evacuated to $10 \mathrm{~Pa}$ and sealed. Samples were then heated at $623 \mathrm{~K}$ for $24 \mathrm{~h}$, and occasionally shaken to ensure homogeneity. Phase purity of the stage- 2 intercalation compound was checked by powder XRD using $\mathrm{Cu} K \alpha$ radiation. In the XRD pattern, the [002] and [003] reflections of the stage-2 compound are the dominant features. Small peaks from the stage-3 compound are also present, containing less than $7 \%$ of the total intensity. Microscopic mixing of stage- 2 and stage- 3 phases has been noted in previous diffraction studies of $\mathrm{KC}_{24}{ }^{12}$ Since stage- 2 and stage- 3 differ only in the sequence of the intercalate layers, this is not expected to affect the in-plane diffusion of adsorbed molecules.

For isotherm measurements, a $0.787 \mathrm{~g}$ sample was transferred to a stainless steel reactor in an argon glovebox. Hydrogen adsorption isotherms were measured at 77, 87, and $195 \mathrm{~K}$ with a custom-built Sieverts apparatus using a standard volumetric measurement procedure. The normal hydrogen equation of state in the REFPROP (Ref. 24) Standard Reference Database was used to calculate the real gas density from the measured equilibrium temperature and pressure.

Neutron-scattering measurements were performed on the high-flux backscattering spectrometer (HFBS) (Ref. 25) and disk chopper spectrometer (DCS) (Ref. 26) at the NIST Cen- ter for Neutron Research. For all neutron experiments, samples were transferred in a high-purity helium glovebox to an annular-geometry aluminum sample cell, sealed with an indium $\mathrm{O}$ ring, and mounted on a closed-cycle helium refrigerator. Sample width was chosen for $10 \%$ total scattering. The sample can was connected with a stainless steel capillary line to a gas-handling unit consisting of a reference volume and a high-resolution pressure transducer. Hydrogen loading was performed at $60 \mathrm{~K}$ by filling the reference volume with the precise amount of $\mathrm{H}_{2}$ gas needed for a $\mathrm{KC}_{24}\left(\mathrm{H}_{2}\right)_{1}$ composition, opening the valve to the sample, and monitoring the pressure as it dropped to approximately $0 \mathrm{kPa}$. A KC $\mathrm{KC}_{24}\left(\mathrm{H}_{2}\right)_{2}$ composition was also prepared by the same method for measurement on HFBS. Scattering from $\mathrm{KC}_{24}$ without $\mathrm{H}_{2}$ was measured at 4 and $25 \mathrm{~K}$ on HFBS and at 40 and $60 \mathrm{~K}$ on DCS. The neutron-scattering spectra at the different temperatures were identical within the instrument resolution, so they were summed and used as the background. The HFBS instrument was operated in both the fixed-window and dynamicwindow modes. In the fixed-window mode, the Doppler drive was stopped and only elastic scattering was recorded. A heating rate of $0.5 \mathrm{~K} / \mathrm{s}$ was used for the temperature scan experiments and the total intensity was obtained by summing over all 16 detector banks. In the dynamic-window mode, the $\mathrm{Si}[111]$ crystal monochrometer was operated at $24 \mathrm{~Hz}$, providing a dynamic range of $\pm 17 \mu \mathrm{eV}$, a $Q$ range of $0.25-1.75 \AA^{-1}$, and an energy resolution of $0.85 \mu \mathrm{eV}$ at the elastic peak. The DCS spectra were acquired using an incident-neutron wavelength of $\lambda=6.0 \AA$ with an available $Q$ range of $0.1-2.0 \AA^{-1}$ and an energy resolution of approximately $60 \mu \mathrm{eV}$. The DAVE software package was used for data reduction and peak fitting. ${ }^{27}$

\section{RESULTS}

\section{A. Isotherms}

Adsorption isotherms of $\mathrm{H}_{2} / \mathrm{KC}_{24}$ are presented in Fig. 2 . At low pressure the 77 and $87 \mathrm{~K}$ isotherms are steeply sloped, while at higher pressures the isotherms reach a common plateau capacity of $2.01 \mathrm{~mol} \mathrm{H}_{2} / \mathrm{mol} \mathrm{KC}_{24}(140 \mathrm{cc} / \mathrm{g}$ STP). This is not typically observed in the supercritical isotherms of activated carbons, where the maximum adsorption decreases with temperature. ${ }^{3}$ The $\mathrm{H}_{2} / \mathrm{KC}_{24}$ isotherms exhibit near-ideal Langmuir adsorption behavior beyond the lowpressure region. The dilute region of the isotherm is shown in the inset of Fig. 2. In this region, the isotherm has a concave upward curvature, presumably due to the expansion of the $c$ axis with $\mathrm{H}_{2}$ adsorption. ${ }^{28,29}$

At low coverage, Henry's law gives a linear relationship between the specific surface excess amount and the equilibrium pressure, $n=k_{H} p$. By fitting to a virial expansion,

$$
\ln (n / p)=C_{1}+C_{2} n+C_{3} n^{2}+\cdots,
$$

accurate values of Henry's law constant were obtained at 77 , 87, and $195 \mathrm{~K}$ from the $y$ intercept: $C_{1}=\ln \left(k_{H}\right)$. The differential enthalpy was then calculated from the van't Hoff equation, yielding a zero-coverage adsorption enthalpy of $8.5 \mathrm{~kJ} /$ mol. 

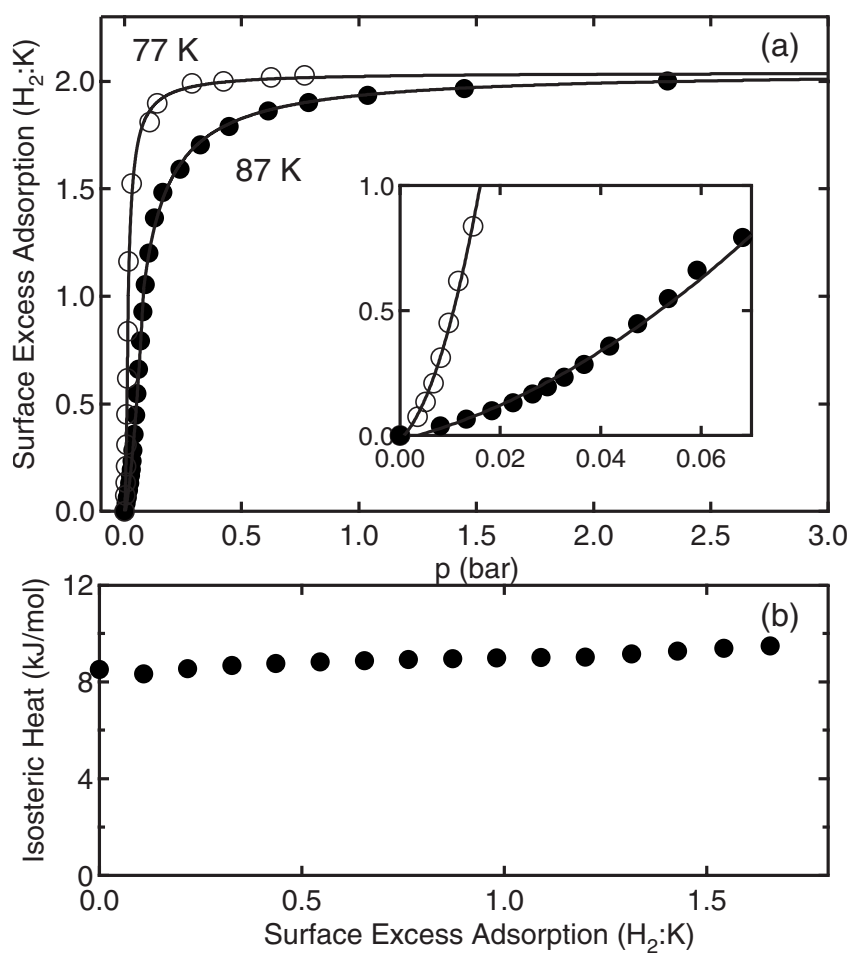

FIG. 2. (a) Volumetric adsorption isotherms of $\mathrm{H}_{2} / \mathrm{KC}_{24}$ measured at 77 and $87 \mathrm{~K}$. The $y$ axis gives the specific surface excess adsorption as a mole ratio of $\mathrm{H}_{2}$ to $\mathrm{K}$. The inset enlarges the lowpressure region of the isotherm. (b) Isosteric enthalpy of adsorption $(\mathrm{kJ} / \mathrm{mol})$ as a function of surface excess adsorption.

The isosteric adsorption enthalpy as a function of surface excess concentration was calculated from the 77 and $87 \mathrm{~K}$ isotherms with the following thermodynamic equation:

$$
\Delta H=R\left[\frac{\partial \ln (p)}{\partial(1 / T)}\right]_{n} .
$$

The natural logarithm of $p$ was plotted against $1 / T$ at constant $n$ to obtain a slope. To obtain consistent values of $p$ as a function of $n$, the isotherms were fitted with an empirical equation-the low-pressure region was fitted to a secondorder polynomial and the remainder was fitted to a Hill equation. The calculated isosteric adsorption enthalpy is displayed in Fig. 2(b). It is nearly constant over most of the adsorption range, with a zero-coverage limit of $8.5 \mathrm{~kJ} / \mathrm{mol}$. The isosteric heat has a slightly positive slope, an anomaly that may originate from the lattice expansion in conjunction with $\mathrm{H}_{2}$ adsorption. 5,29

\section{B. Elastic intensity}

Using the fixed-window mode of operation on HFBS, we measured the elastic intensity of the $\mathrm{H}_{2} / \mathrm{KC}_{24}$ system as a function of temperature. These scans are displayed in Fig. 3. The curve for the control sample of $\mathrm{KC}_{24}$ does not show any major changes between 4 and $100 \mathrm{~K}$. In contrast, the $\mathrm{KC}_{24}\left(\mathrm{H}_{2}\right)_{1}$ and $\mathrm{KC}_{24}\left(\mathrm{H}_{2}\right)_{2}$ curves show a rapid decrease in the elastic intensity starting at 35 and $60 \mathrm{~K}$, respectively. The sudden drop in elastic signal is accompanied by the growth
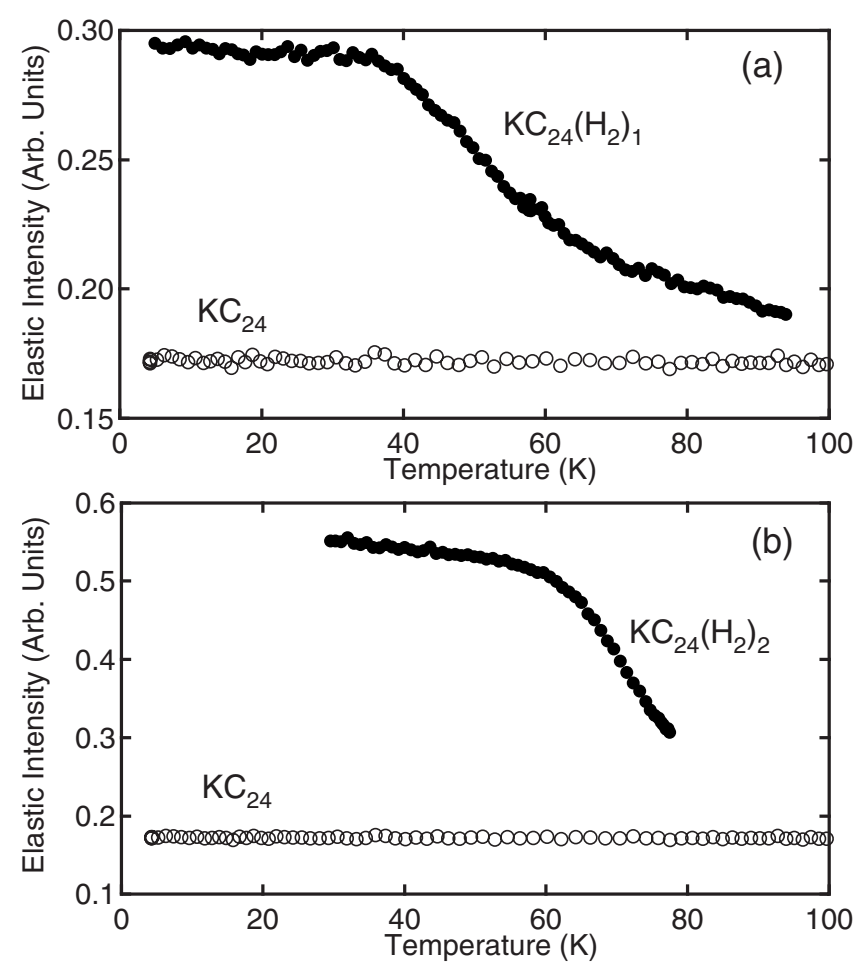

FIG. 3. Elastic intensities from (a) $\mathrm{KC}_{24}\left(\mathrm{H}_{2}\right)_{1}$ and (b) $\mathrm{KC}_{24}\left(\mathrm{H}_{2}\right)_{2}$ as functions of temperature. Intensity from the reference material $\mathrm{KC}_{24}$ is also presented.

in quasielastic intensity (see Fig. 4), indicating the presence of a different phase in which there is hydrogen diffusion. This is discussed in Sec. $\mathrm{V}$ as a melting of the $\mathrm{H}_{2}$ adsorbate that extends over a range of temperatures. Scans obtained from heating and cooling the sample, both at the same rate, did not have any significant differences except that the $\mathrm{KC}_{24}\left(\mathrm{H}_{2}\right)_{1}$ heating curve was slightly more rounded near the transition point at $35 \mathrm{~K}$ compared to the cooling curve.

One possibility that needed to be ruled out was that desorption of $\mathrm{H}_{2}$ could also contribute to the decrease in elastic scattering at higher temperatures. For the composition $\mathrm{KC}_{24}\left(\mathrm{H}_{2}\right)_{1}$ this is unlikely because the equilibrium pressure at $60 \mathrm{~K}$ is close to $0 \mathrm{kPa}$, and the decrease begins at an even lower temperature of $35 \mathrm{~K}$. Nevertheless, to be certain that the elastic intensity was not affected by $\mathrm{H}_{2}$ desorption, we monitored the equilibrium pressure with a high-resolution manometer over the entire temperature scan. We are confident that the concentration of adsorbed $\mathrm{H}_{2}$ did not change significantly over the measured temperature range, and would not have contributed to the large decrease in the elastic intensity. The $\mathrm{KC}_{24}\left(\mathrm{H}_{2}\right)_{2}$ composition was not heated above $80 \mathrm{~K}$ to avoid desorbing the $\mathrm{H}_{2}$ gas.

\section{Quasielastic neutron scattering}

Background-subtracted QENS spectra of $\mathrm{KC}_{24}\left(\mathrm{H}_{2}\right)_{1}$ measured on DCS and on HFBS are shown in Fig. 4. Since the incoherent-scattering cross section of hydrogen is very large, approximately half of the total scattering is from hydrogen. The background signal from $\mathrm{KC}_{24}$ is subtracted to estimate the scattering from hydrogen alone. Some elastic signal due 

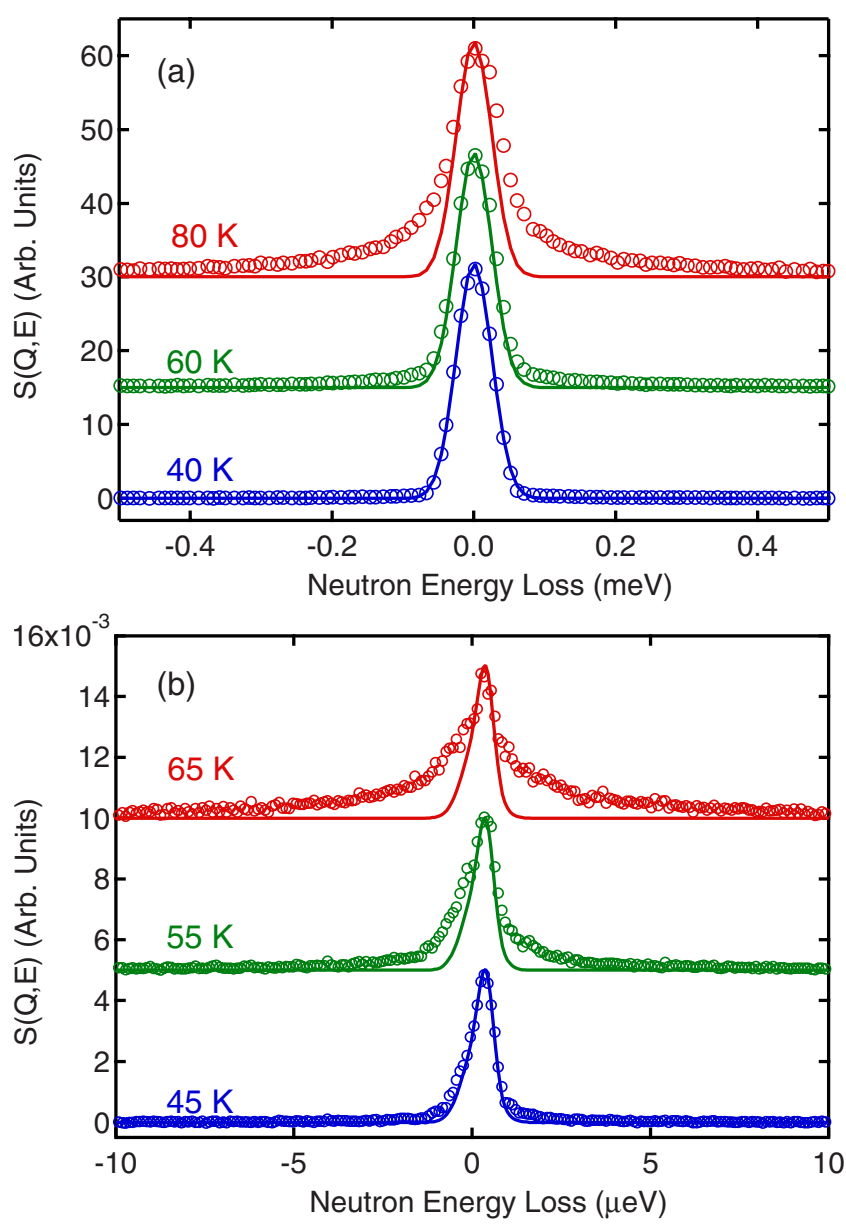

FIG. 4. (Color online) Quasielastic-neutron-scattering spectra of $\mathrm{KC}_{24}\left(\mathrm{H}_{2}\right)_{1}$ : (a) from DCS spectrometer, at $Q=1.84 \AA^{-1}$; (b) from HFBS spectrometer, at $Q=0.87 \AA^{-1}$. The instrument resolution is included for comparison to the spectra at each temperature. The experimental spectra have been scaled to equal height, and offset for clarity.

to scattering from the rigid graphite framework might still remain in the background-subtracted signal, however. Diffraction peaks from the host $\mathrm{KC}_{24}$ material are a significant feature in the $Q$-dependent intensity. Because the metalcontaining graphite galleries expand approximately $5 \%$ upon $\mathrm{H}_{2}$ adsorption, the $\mathrm{KC}_{24}$ diffraction peaks shift to slightly lower $Q$, causing the $\mathrm{KC}_{24}$ background to oversubtract on the high- $Q$ side. Therefore it is necessary to mask the detector banks containing and immediately surrounding the large $\mathrm{KC}_{24}$ diffraction peaks at $Q=0.72 \AA^{-1}$ and $Q=1.45 \AA^{-1}$.

In Fig. 4(a) the DCS spectra show resolution-limited elastic scattering at $40 \mathrm{~K}$ and quasielastic line broadening at higher temperatures. The HFBS spectra displayed in Fig. 4(b) are sensitive to slow processes with characteristic times between approximately 100 and 10000 ps (not resolvable on DCS). Here elastic scattering is dominant at $45 \mathrm{~K}$, while large quasielastic broadening is present at 55 and $65 \mathrm{~K}$. The combination of a sharp elasticlike component and a broad quasielastic component is present in both the DCS and HFBS spectra. The peak intensity decreases with temperature, but in Fig. 4 the spectra have been scaled to equal height to facilitate comparison. Quasielastic scattering is found at the

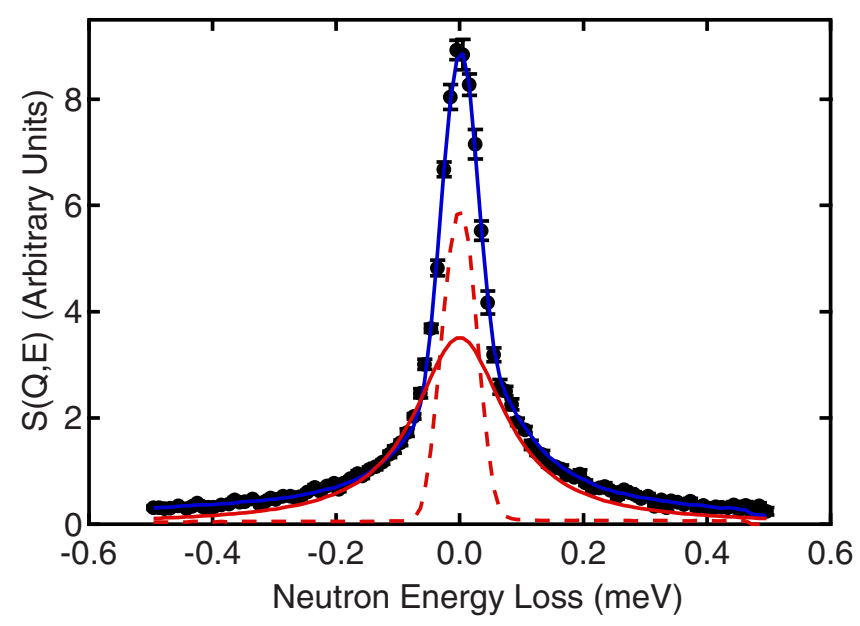

FIG. 5. (Color online) Experimental QENS spectrum of $\mathrm{KC}_{24}\left(\mathrm{H}_{2}\right)_{1}$ measured on DCS at $80 \mathrm{~K}$, with wave vector $Q$ $=1.88 \AA^{-1}$. Experimental points: 0 . Fitted curves: quasielastic (red, solid line), elastic (red, dashed line), and total (blue, solid line). The quasielastic curve is a Lorentzian (before convolution) and the elastic curve is a delta function (after convolution).

same temperatures for Figs. 4(a) and 4(b), but the energy scales are vastly different. This means that there are two diffusion processes present: a fast one and a slow one.

\section{MODELS}

Considering the strength of the $\mathrm{H}_{2}$ adsorption potential in $\mathrm{KC}_{24}$, it is likely that classical jump diffusion is the dominant mechanism for diffusion at these temperatures. Further, it is assumed that the $\mathrm{H}_{2}$ molecules do not cross through the graphite basal planes, but move within the two parallel planes. Because the in-plane alkali-metal structure is not well understood, however, a rigorous jump diffusion mechanism for the $\mathrm{H}_{2} / \mathrm{KC}_{24}$ system cannot be fully developed.

For jump diffusion models the average quasielastic linewidth in the limit of large $Q$ is approximately independent of the geometry of the system, depending only on the characteristic residence times $\tau$ between jumps. The diffusion times can be estimated from the quasielastic spectra at large $Q$ using the relation $\langle\Gamma\rangle=\hbar / \tau$, where $\Gamma$ is the half-width at half maximum (HWHM) of a Lorentzian. To estimate the linewidths at large $Q$, a resolution-broadened scattering function consisting of a Lorentzian plus a delta function (to account for the elasticlike component) and a constant background was fitted to the experimental spectra. For $80 \mathrm{~K}$ spectra at $Q=1.88 \AA^{-1}$, the fitted Lorentzian and delta function components are displayed in Fig. 5. Values of $\tau$ for both the DCS and HFBS data were obtained from averages of $\Gamma$ between $Q=1.1 \AA$ and $Q=1.9 \AA$, and are listed in Table I. The variation in the residence times with temperature roughly follows an Arrhenius relation $\tau=\tau_{0} \exp \left(E_{a} / T\right)$. For the fast-diffusion process, $\tau_{0}=1.0 \pm 0.1 \mathrm{ps}$ and $E_{a}=156 \pm 5 \mathrm{~K}$. For the slowdiffusion process, $\tau_{0}=21 \pm 2 \mathrm{ps}$ and $E_{a}=189 \pm 5 \mathrm{~K}$.

The scattering function at small $Q$ probes large distances and is highly sensitive to both the dimensionality and characteristic lengths of the diffusion process. Since the 
TABLE I. Characteristic diffusion times $\tau$ (ps), calculated from the half-width at half maximum of Lorentzians' fit to the experimental spectra at large $Q$.

\begin{tabular}{cc}
\hline \hline \multicolumn{3}{c}{ (a) Fast process } \\
\hline Temperature (K) & $\tau(\mathrm{ps})$ \\
\hline 60 & $13.4 \pm 0.2$ \\
70 & $9.4 \pm 0.1$ \\
80 & $7.0 \pm 0.1$ \\
& \\
& (b) Slow process \\
\hline Temperature (K) & $\tau(\mathrm{ps})$ \\
\hline 45 & $1330 \pm 36$ \\
55 & $699 \pm 12$ \\
65 & $372 \pm 7$ \\
\hline
\end{tabular}

intercalate-containing galleries in $\mathrm{KC}_{24}$ are wide enough to accept only a single layer of sorbed $\mathrm{H}_{2}$ molecules, hydrogen diffusion perpendicular to the basal planes can be ignored in a first approximation. A two-dimensional translational diffusion model can therefore be used for the $\mathrm{H}_{2} / \mathrm{KC}_{24}$ system with $D$ referring to the coefficient for diffusion parallel to the basal plane. In the limit of low $Q$, the scattering function for a jump diffusion mechanism reduces to a Lorentzian with a HWHM of $\Gamma=\hbar D Q^{2}$ (i.e., equivalent to continuous randomwalk diffusion). For two-dimensional continuous diffusion in a single crystal, the scattering function is given by

$$
S_{2 \mathrm{D}}(Q)=\frac{1}{\pi} \frac{D(Q \sin \theta)^{2}}{\left[D(Q \sin \theta)^{2}\right]^{2}+\omega^{2}},
$$

where $\theta$ is the angle between the scattering vector $\vec{Q}$ and the normal to the diffusion plane. When $\vec{Q}$ is perpendicular to the plane (e.g., $\theta=0$ ), elastic scattering is obtained. Lorentzians of increasing width are obtained as $\theta$ increases. Since the experimental data were collected from powder samples, the orientational average must be calculated, as discussed by Lechner. ${ }^{30,31}$ The powder-averaged scattering function has a cusplike shape with a logarithmic singularity at $\omega=0$. At finite instrument resolution, quasielastic scattering from a two-dimensional system cannot be easily distinguished from a combination of quasielastic and elastic scatterings from an isotropic three-dimensional system. ${ }^{31}$

The scattering function given by Eq. (3), after orientational averaging and convolution with instrument resolution, was fitted to experimental spectra at low $Q$. Fits to the $65 \mathrm{~K}$ spectra collected on HFBS are displayed in Fig. 6. Chisquare values between 1.3 and 2.6 were obtained for fits to the HFBS spectra taken at $55 \mathrm{~K}$ and $65 \mathrm{~K}$, while larger chi-square values between 1.6 and 8.7 were obtained for fits to $80 \mathrm{~K}$ DCS spectra. Fits to lower-temperature data were poor due to the small amount of quasielastic broadening at those conditions. For comparison, low- $Q$ spectra were also fitted to an isotropic three-dimensional diffusion model consisting of a Lorentzian plus a delta function, broadened by

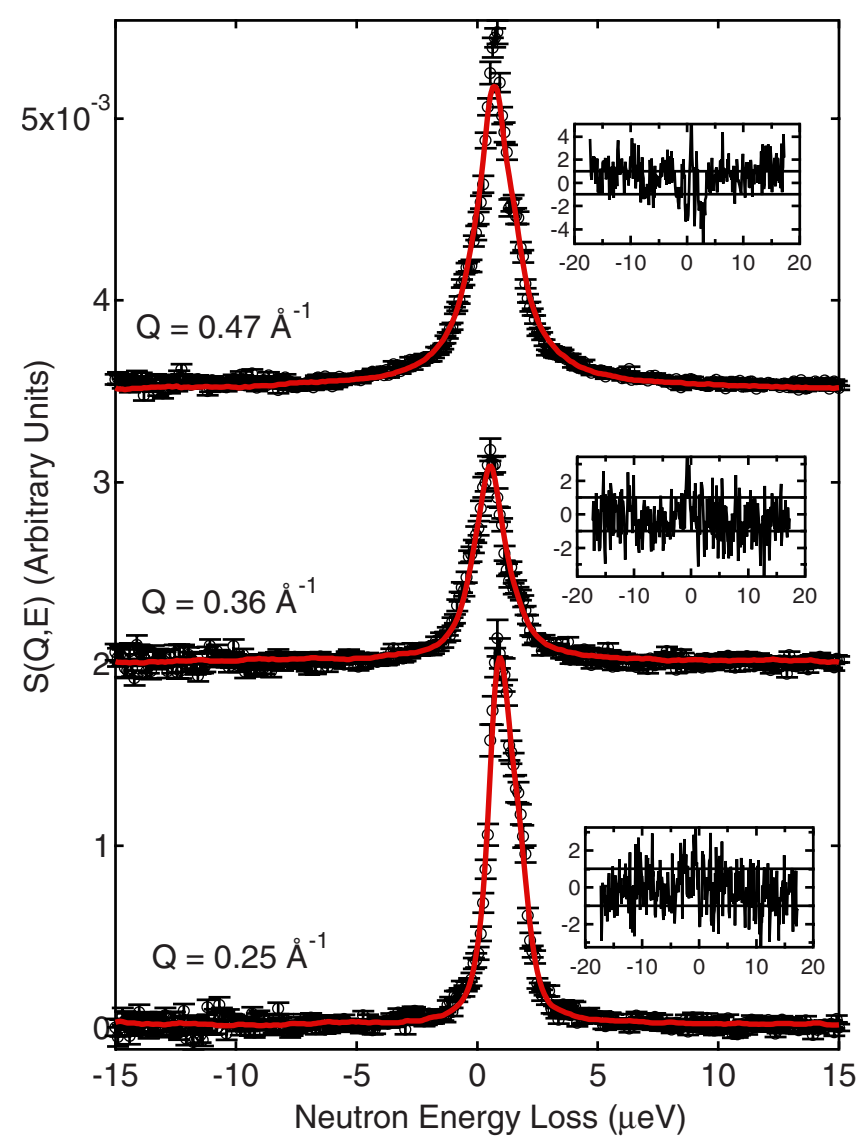

FIG. 6. (Color online) Fits of the incoherent-scattering function for two-dimensional continuous random-walk diffusion to the QENS spectra at low $Q$. Insets show the residuals in units of the standard deviation. Experimental spectra were acquired on the HFBS spectrometer at $65 \mathrm{~K}$.

instrument resolution. This model assumes that one population of $\mathrm{H}_{2}$ molecules is diffusing, while a second population is nondiffusing. Unfortunately, fits to the three-dimensional model produced equivalent chi-square values as the twodimensional model and did not provide a method of distinguishing between the two geometries.

\section{DISCUSSION}

A melting transition was observed for sorbed $\mathrm{H}_{2}$ in the second-stage potassium-graphite intercalation compound $\mathrm{KC}_{24}$. This transition begins in $\mathrm{KC}_{24}\left(\mathrm{H}_{2}\right)_{1}$ at approximately $35 \mathrm{~K}$, compared to $14 \mathrm{~K}$ for the melting of solid $\mathrm{H}_{2}$. The suppression of melting reflects the fact that strong adsorption potentials are present in $\mathrm{KC}_{24}$. (Melting of the potassium superstructure in $\mathrm{KC}_{24}$ occurs at approximately $200 \mathrm{~K}$ and is thought to extend over a wide temperature range. ${ }^{32}$ ) Both elasticlike and quasielasticlike scatterings are present above $35 \mathrm{~K}$, but it is not clear if the elastic scattering is due to a residual solid $\mathrm{H}_{2}$ phase, if it is an artifact of two-dimensional translational diffusion, or if it is unsubtracted background signal from the rigid carbon framework.

A unique feature observed in the $\mathrm{H}_{2} / \mathrm{KC}_{24}$ system is the presence of diffusion processes on two distinct time scales. 
One simple explanation is that the fast process is from the rapid individual jumps of the $\mathrm{H}_{2}$ molecules, whereas the slow process originates from in-plane jumps of the potassium atoms. Potassium atoms are surrounded by a large electron density, and $\mathrm{H}_{2}$ molecules tend to cluster around them due to van der Waals interactions. When a potassium atom jumps, the fast-moving $\mathrm{H}_{2}$ molecules quickly reconfigure around the new sites of the potassium atom. This fluctuation in $\mathrm{H}_{2}$ particle density can be detected by the backscattering spectrometer as slow diffusion. An alternative theory can be developed by noting that diffusion rates may also depend on local concentration. The composition $\mathrm{KC}_{24}\left(\mathrm{H}_{2}\right)_{1}$ is not saturated, and self-interactions between sorbed $\mathrm{H}_{2}$ molecules may cause them to coalesce into tightly packed islands. Molecules in the interior of these islands will have far fewer neighboring vacancy sites compared to molecules on the cloud periphery and will have slower diffusion rates. Measurements on the $\mathrm{KC}_{24}\left(\mathrm{H}_{2}\right)_{2}$ composition are needed to verify the $\mathrm{H}_{2}$ diffusion behavior in a tightly packed arrangement.

Because the diameter of $\mathrm{H}_{2}$ is similar to the gap between intercalated planes in $\mathrm{KC}_{24}$, it would appear at first glance that there is little room for translational motion perpendicular to the basal plane. Molecular-dynamics simulations of $\mathrm{KC}_{24}\left(\mathrm{H}_{2}\right)_{2}$ at $T=500 \mathrm{~K}$, however, indicate that perpendicular $\mathrm{H}_{2}$ motions are present in the system, and that $\mathrm{H}_{2}$ motion is essentially random within a $1.4 \AA$ lamellar region centered between the graphite sheets. ${ }^{6}$ The incoherent-scattering function for random-walk diffusion within a lamellar region is given by an infinite sum of Lorentzians with $Q$-dependent amplitudes, though this sum can be truncated after five terms for our $Q$ range. ${ }^{33}$ Since it was not possible to distinguish even between ideal two-dimensional and three-dimensional diffusion models, however, fitting to a lamellar diffusion model will not yield any significant information from the low- $Q$ data. Higher-quality QENS spectra from a singlecrystal $\mathrm{KC}_{24}$ sample are probably required to rigorously sort out the dimensionality of the $\mathrm{H}_{2}$ dynamics.

An additional topic of interest concerns the origin of the elasticlike peaks in the QENS spectra measured on both DCS and HFBS. While elasticlike features have been observed in the QENS spectra of $\mathrm{H}_{2}$ adsorbed in zeolites and carbon nanostructures, they have typically been explained in terms of nondiffusing $\mathrm{H}_{2}$ populations. ${ }^{20-23}$ Such a scenario can also apply to $\mathrm{KC}_{24}$ if the $\mathrm{H}_{2}$ molecules form islands, suppressing the diffusion rates of molecules trapped in their interiors. Quantum delocalization within a single sorption site, as illustrated in Fig. 1, may also complicate the QENS spectra. However, a characteristic of spatially bounded diffusion is the presence of an elastic incoherent structure factor similar to that observed in rotational diffusion. ${ }^{33}$ Rotational diffusion, though, can be ruled out as a source of quasielastic broadening in our QENS spectra because transitions between rotational states (which have large energies compared to translations) occur on a much faster time scale than can be measured on DCS or HFBS.

In the phase diagram of $\mathrm{H}_{2}$ monolayers on pristine graphite, transitions between commensurate and incommensurate solid 2D phases are well documented. ${ }^{34}$ As the surface coverage is increased and the $\mathrm{H}_{2}-\mathrm{H}_{2}$ interactions become dominant, the commensurate $2 \mathrm{D}$ solid monolayer becomes unstable relative to the formation of dislocations, leading to a domain-wall intermediate phase. Finally, at higher temperatures an isotropic 2D liquid is formed. The transition from a solid to a liquid phase occurs at around $20 \mathrm{~K}$. Although it is tempting to compare melting in the $\mathrm{KC}_{24}\left(\mathrm{H}_{2}\right)_{1}$ system to a Kosterlitz-Thouless-type 2D phase transition, which is marked by the spontaneous formation of dislocations (domain walls) in a solidlike $\mathrm{H}_{2}$ phase, ${ }^{35}$ the experimental evidence presented here is not yet sufficient to support such a comparison.

\section{CONCLUSION}

We studied the sorption enthalpy and diffusion of molecular hydrogen in $\mathrm{KC}_{24}$. The isosteric enthalpy is nearly constant as a function of excess adsorption, with a zero-coverage value of $8.5 \mathrm{~kJ} / \mathrm{mol}$. In neutron-scattering experiments, we observe a strong decrease in elastic scattering beginning at $35 \mathrm{~K}$ in the $\mathrm{KC}_{24}\left(\mathrm{H}_{2}\right)_{1}$ sample, which is attributed to a melting transition. Quasielastic scattering is observed from both a slow-diffusion process and a fast-diffusion process which coexist above $35 \mathrm{~K}$. These follow an Arrhenius relationship $\tau$ $=\tau_{0} \exp \left(E_{a} / T\right)$, with $\tau_{0}^{\text {fast }}=1.0 \pm 0.1 \mathrm{ps}, \quad \tau_{0}^{\text {slow }}=21 \pm 2 \mathrm{ps}$, $E_{a}^{\text {fast }}=156 \pm 5 \mathrm{~K}$, and $E_{a}^{\text {slow }}=189 \pm 5 \mathrm{~K}$. The fast-diffusion process is attributed to individual motions of $\mathrm{H}_{2}$ molecules on the time frame of a static potassium structure. The slowdiffusion process is tentatively attributed to fluctuations in $\mathrm{H}_{2}$ particle density correlated with jumps of potassium atoms.

\section{ACKNOWLEDGMENTS}

This work utilized facilities supported in part by the National Science Foundation under Agreement No. DMR0454672 and was partially supported by the Office of Energy Efficiency and Renewable Energy through the Hydrogen Sorption Center of Excellence under Contract No. DE-FC36$05 \mathrm{GO} 15079$.

\footnotetext{
*purewal@caltech.edu

${ }^{\dagger}$ Also at Department of Materials Science and Engineering, University of Maryland, College Park, MD, USA.

${ }^{1}$ S. K. Bhatia and A. L. Myers, Langmuir 22, 1688 (2006).

${ }^{2}$ A. Züttel, Naturwiss. 91, 157 (2004).
}

${ }^{3}$ R. Ströbel, J. Garche, P. T. Moseley, L. Jörissen, and G. Wolf, J. Power Sources 159, 781 (2006).

${ }^{4}$ K. Watanabe, T. Kondow, M. Soma, T. Onishi, and K. Tamaru, Proc. R. Soc. London, Ser. A 333, 51 (1973).

${ }^{5}$ P. Lagrange, D. Guerard, J. F. Mareche, and A. Herold, J. Less- 
Common Met. 131, 371 (1987).

${ }^{6}$ H. Cheng, G. Pez, G. Kern, G. Kresse, and J. Hafner, J. Phys. Chem. B 105, 736 (2001)

${ }^{7}$ T. Enoki, S. Miyajima, M. Sano, and H. Inokuchi, J. Mater. Res. 5, 435 (1990).

${ }^{8}$ I. Cabria, M. J. Lopez, and J. A. Alonso, J. Chem. Phys. 123, 204721 (2005).

${ }^{9}$ W.-Q. Deng, X. Xu, and W. A. Goddard, Phys. Rev. Lett. 92, 166103 (2004).

${ }^{10}$ G. L. Doll and P. C. Eklund, Phys. Rev. B 36, 9191 (1987).

${ }^{11}$ S. B. DiCenzo, Phys. Rev. B 26, 5878 (1982).

${ }^{12}$ F. Rousseaux, R. Moret, D. Guerard, and P. Lagrange, Phys. Rev. B 42, 725 (1990).

${ }^{13}$ G. R. S. Naylor and J. W. White, J. Chem. Soc., Faraday Trans. 1 83, 3447 (1987).

${ }^{14}$ R. Clarke, J. N. Gray, H. Homma, and M. J. Winokur, Phys. Rev. Lett. 47, 1407 (1981).

${ }^{15}$ A. Lovell, F. Fernandez-Alonso, N. T. Skipper, K. Refson, S. M. Bennington, and S. F. Parker, Phys. Rev. Lett. 101, 126101 (2008).

${ }^{16}$ J. P. Beaufils, T. Crowley, T. Rayment, R. K. Thomas, and J. W. White, Mol. Phys. 44, 1257 (1981).

${ }^{17}$ W. J. Stead, I. P. Jackson, J. McCaffrey, and J. W. White, J. Chem. Soc., Faraday Trans. 2 84, 16669 (1988).

${ }^{18}$ A. P. Smith, R. Benedek, F. R. Trouw, M. Minkoff, and L. H. Yang, Phys. Rev. B 53, 10187 (1996).

${ }^{19}$ A. Lovell, S. M. Bennington, N. T. Skipper, C. Gejke, H. Thompson, and M. A. Adams, Physica B 385-386, 163 (2006).

${ }^{20}$ F. Fernandez-Alonso, F. J. Bermejo, C. Cabrillo, R. O. Loutfy, and M. L. Saboungi, Phys. Rev. Lett. 98, 215503 (2007).
${ }^{21}$ D. G. Narehood, J. V. Pearce, P. C. Eklund, P. E. Sokol, R. E. Lechner, J. Pieper, J. R. D. Copley, and J. C. Cook, Phys. Rev. B 67, 205409 (2003).

${ }^{22}$ H. Fu, F. Trouw, and P. E. Sokol, J. Low Temp. Phys. 116, 149 (1999).

${ }^{23}$ R. Kahn and E. V. E. C. De Lara, J. Chem. Phys. 91, 5097 (1989).

${ }^{24}$ E. W. Lemmon, M. L. Huber, and M. O. McLinden, NIST Standard Reference Database 23: Reference Fluid Thermodynamic and Transport Properties (REFPROP), version 8.0, in Standard Reference Data Program, National Institute of Standards and Technology, Gaithersburg, 2007.

${ }^{25}$ A. Meyer, R. M. Dimeo, P. M. Gehring, and D. A. Neumann, Rev. Sci. Instrum. 74, 2759 (2003).

${ }^{26}$ J. Copley and J. Cook, Chem. Phys. 292, 477 (2003).

${ }^{27}$ http://www.ncnr.nist.gov/dave

${ }^{28}$ G. L. Doll, P. C. Eklund, and G. Senatore, in Intercalation in Layered Materials, edited by M. S. Dresselhaus (Plenum, New York, 1986), p. 309.

${ }^{29}$ S. A. Solin and H. Zabel, Adv. Phys. 37, 87 (1988).

${ }^{30}$ A. J. Dianoux and F. Volino, Mol. Phys. 30, 1181 (1975).

${ }^{31}$ R. E. Lechner, Solid State Ionics 77, 280 (1995).

${ }^{32}$ H. Zabel, A. Magerl, J. J. Rush, and M. E. Misenheimer, Phys. Rev. B 40, 7616 (1989).

${ }^{33}$ P. L. Hall and D. K. Ross, Mol. Phys. 36, 1549 (1978).

${ }^{34}$ H. Freimuth, H. Wiechert, and H. J. Lauter, Surf. Sci. 189-190, 548 (1987).

${ }^{35}$ S. N. Coppersmith, D. S. Fisher, B. I. Halperin, P. A. Lee, and W. F. Brinkman, Phys. Rev. B 25, 349 (1982). 